\title{
Research on Soil Fertility Information Collection System Based on Wireless Sensor Network
}

\author{
Duan Yunpeng \\ School of information technology, Jilin Agricultural University, Changchun, China \\ duanyunpengjoan@sohu.com
}

Keywords: Data acquisition; wireless sensor networks; soil nutrients; ZigBee; 4G

\begin{abstract}
The collection system of soil fertility data is to collect the soil fertility information of agriculture by wireless sensor network, so as to provide data support for modern agriculture. With the precision agriculture, the computer technology is more and more applied to agricultural production, and the data of soil fertility is applied to the soil fertility data by wireless sensor network technology. According to the effective data analysis, the nutrients needed in the soil are obtained, and then the scientific ratio is carried out, a more efficient ratio scheme is obtained, and the scientific and rational fertilization can not only improve the fertilization level, but also improve the efficiency of the work, thus greatly reducing the production cost of farmers, protecting the soil, reducing pollution and improving the quality and safety of agricultural products
\end{abstract}

\section{Introduction}

The phenomenon of blind and confused application of chemical fertilizer in our country's agricultural production and life has not only resulted in waste of fertilizer, but also resulted in the decline of production quality of agricultural products and environmental pollution, which seriously affected the stability and improvement of agricultural productivity. In view of the above problems, through the long-term research and practice of experts and scholars at home and abroad, the technology of soil testing and formulated fertilization is put forward. Soil testing and formula fertilization technology is the application of modern agricultural research fertilization technology. On the basis of soil testing and field test, the technology of fertilizer application of nitrogen, phosphorus, potassium and medium and trace elements was put forward on the basis of the amount of fertilizer, the ability of soil fertilizer and the effect of fertilizer. This technology can give full play to the potential of cultivated land and achieve high quality and high yield of crops, and it is of great significance to save land resources, protect the agricultural ecological environment and accelerate the development of modern agriculture in China. Jilin province is an important commodity grain production base of the country. Corn is one of the important crops in Jilin province. This information collection system uses wireless sensor network to collect the mobile information acquisition equipment in a certain period of time, which makes the difficulty of data collection greatly reduced, 
and also reduces the related cost, through PC, Web site, touch screen, smart phone, WeChat platform and other channels, let users get the soil temperature, humidity, dissolved oxygen content, $\mathrm{pH}$ value and nitrogen, phosphorus, potassium and other related information.

\section{System overall design scheme}

The design of soil fertility information collection system based on wireless sensor network is based on the project of scientific research, which is mainly composed of 3 parts, which are data collection, data transmission and data monitoring, which are composed of 3 parts $[1,6]$. Data collection mainly realizes the automatic collection function of soil nutrient data. The data collected need a wide range of monitoring, monitoring accuracy, high monitoring stability and so on. The wireless transmission of data uses $4 \mathrm{G}$ wireless mobile communication technology to combine low power and ZigBee technology with self-organizing ability to build wireless sensor network, so as to realize the no docking of farmland information with mobile communication network. Remote monitoring of data is a remote monitoring center based on Web technology. Users do not need to install specific monitoring software, only in the area where there is a network; the information can be viewed through the browser of the mobile phone or computer [2]. As shown in Figure 1.

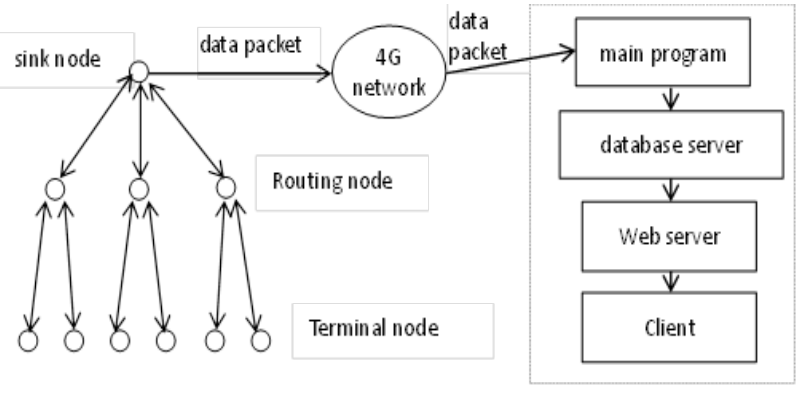

Fig.1 system structure diagram

\section{System hardware design}

\subsection{Soil fertility sensor}

With the rapid development of agriculture, it is necessary to improve the fertility of soil by measuring soil and fertilizer in order to increase the yield and increase the harvest. The traditional method of soil sampling and fertilization cannot meet the needs of modern agricultural development, especially in the application scenes of soil, water and fertilizer, and the rapid change of light. The traditional soil sampling and analysis takes a long time and the accuracy of measurement is influenced by human factors. Therefore, the effect of soil fertility cannot be improved [3]. With the rapid development of the Internet of things technology, it is only through on-line measurement of the Internet of things to measure the parameters of the soil in real time, so that the effect of soil fertility can be improved [5] better. As shown in Figure 2.

At present, the data collected by sensors used to measure soil parameters are relatively simple; often a sensor can collect only one kind of data. If users need to collect a variety of data at the same time, many sensors must be purchased and deployed at the same time. On the one hand, the user needs a lot of time and energy in the selection of products, and the use of a variety of sensors will inevitably lead to a great increase in the time and human cost of daily maintenance. On the other hand, when the system is docking with various sensors, there may be various adaptable problems or conflicts, and cannot meet the needs of the development of Agricultural Internet of things. 


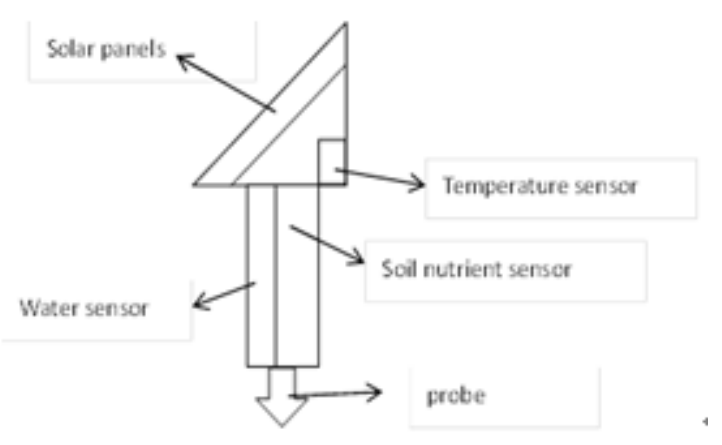

Fig.2 Structure map of soil nutrient sensor

The soil fertility measurement sensor integrates the temperature and humidity sensors, the light sensor, the soil nutrient sensor, the mobile communication module, the positioning module and the communication module, so that the sensor can detect several soil parameters, such as temperature, humidity, light and soil nutrients, so that the sensor can collect and use the sensor in real time. The collection of soil, air and data, without the need for users to deploy additional wireless network rings, and upload clouds through communication modules, combined with cloud data collection and system analysis, requires a single sensor to provide stable and continuous quantitative soil planting environment, sunlight, and water analysis [4]. In addition, the sensor also has built-in solar rechargeable battery to increase endurance, and ensure that the sensor can run in the normal field environment for a long time. Therefore, the utility model effectively overcomes all kinds of shortcomings in the existing technology and has high industrial value.

\subsection{Wind speed sensors}

In the process of wind speed detection, the three-cup sensor is the most widely used. The threecup wind speed sensor consists of three classic low-inertial hemispherical air cups that form 120 degrees each other and are fixed on a rotating shaft. Its working principle is that the sensor has a photoelectric conversion codeplate inside. When there is wind blowing, the wind cup drives the rotating shaft, so that the conversion codeplate converts the wind speed into a photoelectric signal and outputs the wind speed by pulse counting. This system uses QS-FS wind speed sensor, which is small and convenient to carry, adopts flange installation method, and is convenient for installation. The sensor adopts high quality aluminum alloy as raw material, which has the characteristics of good corrosion protection and anti-erosion, and can ensure that it can not be rusted in outdoor use for a long time.

\subsection{Wind direction sensors}

Wind direction usually refers to the near-ground wind direction of the field farmland environment. The detection methods are photoelectric, voltage-type and Romanesque. The photoelectric wind sensor is the most commonly used. It consists of a wind arrow fixed to a rotating axis with a photograyer disk. The arrow of the sensor senses the wind direction information of the outside world and transmits it to the internal code plate. According to the photoelectric signal conversion principle, the corresponding wind direction information is output. The QS-FX 360-degree wind sensor is selected in this paper. It is compact and light in appearance, beautiful and strong, easy to carry and assemble, and accurate in information collection. It mainly uses high-quality aluminum alloy as raw material, which has the characteristics of good corrosion protection and anti-erosion, and can be used 
for the detection of farmland environment in the wild for a long time.

\subsection{Solar power supply module}

The soil fertility information collection system needs to work in the wild for a long time, and the environmental facilities are relatively poor. It is extremely inconvenient to replace batteries frequently because of dry battery power supply. Solar energy is a renewable and widely distributed clean energy. At present, it has been widely used in the field to provide power for wireless sensor nodes. The process of solar energy application usually consists of three parts: solar panel, solar charge and discharge controller and battery. Solar panels absorb solar energy and produce photochemical reactions, converting light energy into electrical energy, which provides energy storage for batteries. The solar charge and discharge controller can ensure that the battery performs charging and discharging in the best way, and can extend the battery's power supply and service life. In the process of designing the solar power supply system, the power supply voltage and current requirements and energy loss of each sensor node should be fully considered. Then the solar battery board, battery selection and solar charge discharge control circuit should be designed. As shown in Figure 3.

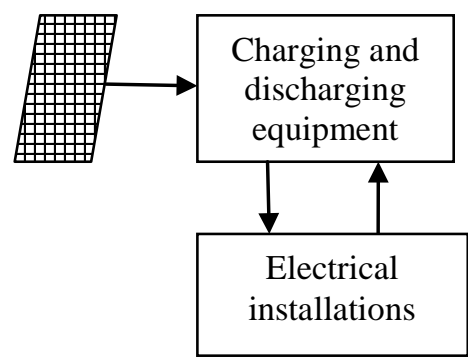

Fig.3 Solar System Framer

\subsection{Terminal design}

Data monitoring is mainly composed of main service program, database server, Web server and client browser. The main service program is responsible for the connection of the TCP/IP packets packaged by the aggregation node to the database server. The data packets are checked and the useful packets are stored in the database, and the incomplete or incorrect data packets are removed from the [7]. The Web server, as the intermediate bridge between the client end browser and the database server, extracts the data from the database and provides the browser to display through the corresponding web script information. The client browser can realize the functions of displaying, querying and downloading all kinds of data collected from farmland. As shown in Figure 4.

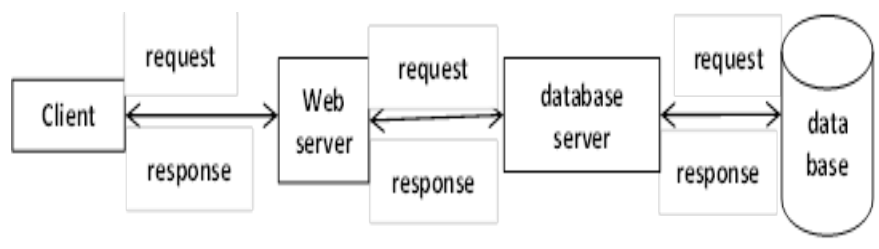

Fig.4 Data processing architecture diagram 


\section{Key technology}

\subsection{Data fusion technology}

TINA is a mechanism that uses sensor nodes to collect data in time for consistency in the network. It meets the needs of users for data accuracy, and saves energy as much as possible through integration within the network. The basic idea is that only when the difference between the data collected and the data collected on the previous one is greater than the tolerance limit specified by a certain user, the node can send the data. It uses directional diffusion to establish routing trees, assigning gradient values to each node and assigning its parent nodes. In order to utilize the time consistency of data, nodes must store additional information. Leaf nodes need to save data sent to the parent node at the last time. The forwarding node not only needs to save the data that it sent to the parent node at the last time, but also needs to save the latest data from each sub node.

\subsection{Data coverage technology}

The coverage control technology of wireless sensor networks is to achieve the purpose of collecting and managing information in the monitoring area through the collaboration of sensor nodes. In the case of the limited capacity of the sensor nodes, such as their own energy, communication bandwidth, and network computing and processing capability, the wireless sensor network can dynamically configure the node parameters such as node location and node radius. Finally, the $\mathrm{W}$ realizes the optimal allocation of various sources of the wireless sensor network, and makes the sensing and monitoring of the sensor nodes. The service quality of sensing and communication has been improved. The system uses coverage type as deterministic coverage and improves network coverage based on improved step size Drosophila optimization algorithm

\section{System test}

\subsection{Node communication distance test}

The transmitting energy, antenna height and surrounding environment will affect the distance of node communication. The greater the transmitting power of nodes, the farther the communication distance is. The system tests the effective communication distance on the flat and sloping fields by changing the transmitting power of nodes. The height of the sink antenna is 5 meters, and the sub node is 3 meters, which is higher than that of the corn plant. The signal is sent to the sink node, and the LED lamp flashing of the sink node is used as the normal communication indication, and the transmission power [6] of the sub node is changed. As shown in Table 1.

Table 1. Emissivity and distance relation table

\begin{tabular}{cccccc}
\hline $\begin{array}{c}\text { Emissive } \\
\text { power }\end{array}$ & $\begin{array}{c}- \\
10 \mathrm{dBm}\end{array}$ & $-5 \mathrm{dBm}$ & $0 \mathrm{dBm}$ & $5 \mathrm{dBm}$ & $10 \mathrm{dBm}$ \\
\hline $\begin{array}{c}\text { Flat } \\
\text { ground }\end{array}$ & 58 & 53 & 58 & 72 & 91 \\
$\begin{array}{c}\text { Sloping } \\
\text { fields }\end{array}$ & 47 & 48 & 52 & 65 & 86 \\
\hline
\end{tabular}




\subsection{Effective packet rate test}

According to the hardware and software designed by the system, 9 nodes are divided into two groups, one of the 9 coordinators $(00)$, two router nodes $(04,14)$, and 6 terminal 5 terminal nodes $(01$, $02,03,11,12$, etc.), and the communication distance between the nodes and the routing nodes. For 50m, 02 and 12 are 40m, 03 and 11 are 40m, 04 and 04, and the distance from sink nodes is 30m. The nodes send data once every 10 minutes, and carry out statistical analysis for 7 days of communication packets. The effective packet rate is shown in Table 2.

Table 2. Effective transmission packet ratio

\begin{tabular}{cccc}
\hline Node label & Send packets & Receive packets & Effective transmission packet ratio \\
\hline 01 & 1264 & 1228 & $97.15 \%$ \\
02 & 1264 & 1221 & $96.60 \%$ \\
03 & 1264 & 1238 & $97.94 \%$ \\
04 & 4901 & 4846 & $98.88 \%$ \\
11 & 1264 & 1259 & $99.60 \%$ \\
12 & 1264 & 1256 & $99.37 \%$ \\
13 & 1264 & 1243 & $98.34 \%$ \\
14 & 4902 & 4876 & $99.47 \%$ \\
\hline
\end{tabular}

\section{Conclusion}

The monitoring of soil environmental information can provide better guidance for formula fertilization. In this system, ZigBee technology, 4G network technology and database technology are used to collect the soil nutrients in the experimental field, fuse the collected data and transmit the data to the user terminal. The user can see the data of soil nutrients in real time or regularly, and the trend of reducing the fertilizer is obtained by analysis. Then the formulation of fertilizer and the best period of fertilization have been made to improve the problem of the present disorderly fertilizer. The real realization of scientific farming can not only guarantee the yield of the crops but also improve the quality of the grain, so that the agricultural ecology can be greatly improved.

\section{Acknowledgment}

This work was financially supported by Jilin Provincial Department of education 13th Five-Year science and technology research project: Research on soil testing and formula fertilization system based on wireless sensor network (JJKH20170295KJ) fund.

\section{References}

[1] Jin Kai Jun, Li Jiang. Data acquisition system based on wireless sensor network. [J]. modern electronic technology, 2017 (40): 72-73.

[2] Zhu Kai, Zhu Huibin, Bai Lizhen, Zhang Jianwei, Xi army, Cao Kegao. ZigBee and C\# based farmland data acquisition system [J]. sensors and Microsystems, 2017 (36): 95-98,

[3] Wang Ren Ran, Pang Junteng, Chen Junjian, Pei Yu, Du Qiliang. Design and implementation of the control system for data acquisition intelligent car based on Arduino control board [J]. Computer technology and automation, 2017 (36): 66-73.

[4] Zuo Gang, Liu Yanchang, Wang Jianping. Based on Arduino and VI, the design of farmland information wireless collection system [J]. Agricultural Mechanization Research, 2016 (2): 213-217.

[5] Zhang Zenglin, Yu Xiaoqing. Soil information acquisition system based on wireless sensor network [J]. Energy saving irrigation, 2011 (12): 41-43.

[6] Feng Ronghua. Design and implementation of wireless sensor network monitoring system for farmland environment 
[D]. Fujian: Fujian Agriculture and Forestry University, 51-52.

[7] Sun Yuwen. Research and implementation of farmland environmental monitoring system based on wireless sensor network [D]. Nanjing: Nanjing Agricultural University, 2013,7-9.

[8] Liu Zhaorong. Based on mobile GIS, the high resolution soil fertility information acquisition system in county area [J]. Agricultural Mechanization Research, 2015 (3): 222-225.

[9] Wang Caifen. Construction and application of soil nutrient information system in County orchard based on WEBGIS [D]. Xianyang: Northwest Agriculture and Forestry University, 2016 (5).45-50

[10] Li Guanfeng, Chen Dongmei. Design of Farmland Monitoring System Based on ZigBee technology [J]. Agricultural Mechanization Research, 2013, 35 (11): 107 -10.

[11] Guo Jia, Ma Xinming, Guo Wei, et al. Design of farmland information collection system based on Zig Bee network [J]. Agricultural Mechanization Research, 2013, 35 (11): 65 - 70. 